\title{
Fast Magnetic Measurements Of 8.6 m Undulator
}

\author{
I. V. Davidyuk ${ }^{1,2}$, Ya. I. Gorbachev ${ }^{1}$, O. A. Shevchenko ${ }^{1}$ \\ ${ }^{1}$ Budker INP SB RAS, Novosibirsk, Russia, daveduke@ outlook.com \\ ${ }^{2}$ Novosibirsk State University, Novosibirsk, Russia
}

\begin{abstract}
The design of the large-aperture variable-period undulator (VPU) developed for the Novosibirsk free electron laser (FEL) is shortly described. High amplitude of on-axis field is achieved due to the arc shape of the magnet blocks and poles of the undulator. To conduct magnetic measurements and fine tuning of the undulator as well as to perform real time position tracking of magnet blocks, pulsed wire technique was adopted.
\end{abstract}

\section{Introduction}

A permanent magnet variable-period undulator with large (1.4) ratio of the aperture to the minimum period and possibility to change the number of poles was designed to upgrade the first stage FEL of the Novosibirsk FEL facility. The Novosibirsk FEL is a source of radiation in the $\mathrm{THz}$ range. The new undulator will replace the existing insertion device section which consists of two 4-meter-long undulators and the three-pole buncher. That will shift long-wave border of the tuning range from $200 \mu \mathrm{m}$ to $450 \mu \mathrm{m}$. A comparison of parameters of former and new undulators is given in the following table:

\begin{tabular}{|l|l|l|}
\hline Parameter & EM undulators & VPU \\
\hline Period, $\mathrm{cm}$ & 12 & $10-16$ \\
\hline Gap (inner diameter), $\mathrm{cm}$ & 8 & 14 \\
\hline Number of periods & $2 \times 32$ & $50-80$ \\
\hline Radiation wavelength, $\mu \mathrm{m}$ & $90-240$ & $82-450$ \\
\hline Deflection parameter & $0-1.1$ & $0.45-1.9$ \\
\hline Full length, cm & $2 \times 400$ & 860 \\
\hline
\end{tabular}

The idea of a VPU with free moving poles was proposed in [1]. At the minimum period $l u_{\min }$, the developed undulator is a conventional hybrid device with neodymium magnets and poles made of soft steel with large permeability. All poles in the undulator are split into halves, and so the undulator is two arrays (upper and lower) of separate removable blocks. Each block is $l_{\mathrm{min}} / 2$ long and consists of a magnet embraced by two pole halves and the whole structure is set in an aluminum frame. The pairs of blocks in upper and lower arrays are connected and placed on a carriage that can move freely along the undulator, see Fig. 1. Adjacent magnets in an array always have antiparallel longitudinal magnetizations, so they experience strong repulsion from each other. Due to repulsion force and the low-friction between guide rail and carriages, the undulator period can be changed via shifting the terminal blocks. This procedure, which changes the overall length of the undulator, is performed by pushers that can be remotely controlled. More details about similar undulator can be found in [2].

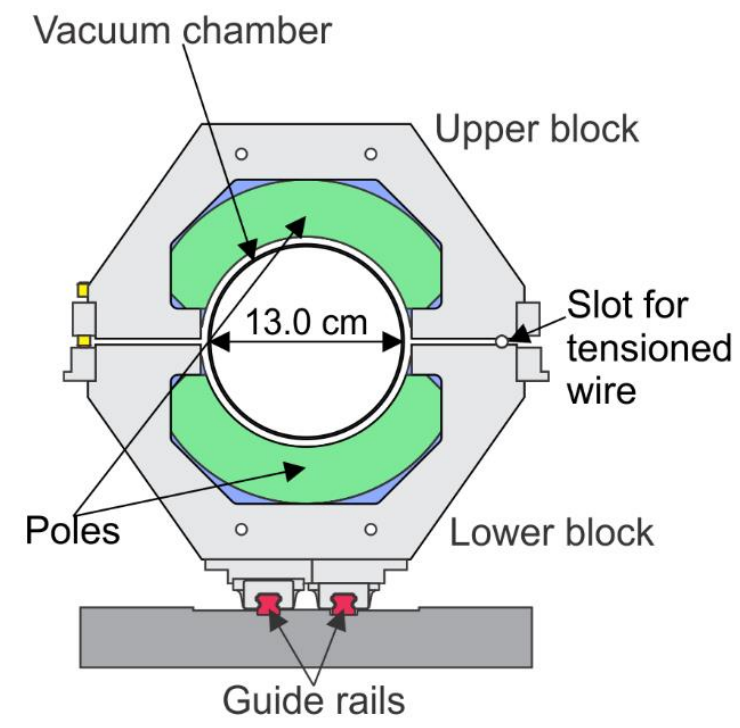

Fig. 1. Front view of the undulator

Obviously blocks in the undulator can create inhomogeneous distribution which may lead to phase errors between the radiation field and the electron transverse motion. The construction of the undulator provides possibility to make in situ measurements of magnetic field distribution in real time to monitor blocks' position errors and calculate the radiation spectrum via pulsed wire method. Slot for the stretched wire in undulator block is shown in Fig. 1.

The pulsed wire technique for magnetic measurements was proposed in [3] and since then it has been used for measurements of field when undulator gap cannot fit the support of the Hall probe or to speedup the tuning of the undulator [4]. This method allows several measurements per second, thus it would significantly reduce the time of magnetic investigation of the developed 8.6 meters long VPU for the first FEL of Novosibirsk FEL facility.

\section{Measurement system}

The basic components of the measurement setup are a wire stretched along the axis of investigated magnetic field, a current pulse generator and a detector of the wire displacement. When current is passing through the wire it interacts with the magnetic field. Flexural waves that travel through the wire towards detector contain information about transverse field profile in the region of interest.

Theoretical analysis of this technique is presented in [5] where the stretched wire is treated as a thin rod in order to take dispersion into account. The expression for wire displacement at the detector position $z=0$ 
reads:

$$
\begin{gathered}
x(0, t)=\frac{1}{2 \sqrt{\mu T}} * \\
* \int_{t-\tau}^{t} I\left(t-t^{\prime}\right) \int_{0}^{v_{0} t^{\prime}} B_{e f f}(z) d z d t^{\prime},
\end{gathered}
$$

where $\mu$ is the mass per unit length of the wire, $T$ is the tension in the wire, $\tau$ is current pulse duration, $v_{0}$ is $\sqrt{T / \mu}$ and $B_{\text {eff }}$ is defined by the following expression:

$$
B_{\text {eff }}(z)=\int_{-\infty}^{\infty} A(k) e^{-i \frac{\omega(k)}{v_{0}} z} d k
$$

where $A(k)$ is the Fourier transform of the magnetic field in the path of the current and $\omega(k)$ is the dispersion relations for the flexural waves in the wire [5]. Using above expressions one can correct effects of dispersion, current pulse form averaging and restore the magnetic field distribution from the data obtained by the detector.

An experimental setup was constructed in order to adopt the technique. A beryllium copper wire with a diameter of $200 \mu \mathrm{m}$ and length of $10 \mathrm{~m}$ was stretched by applying tension close to yield point. A capacitor based current pulse generator can generate pulses with duration of $50 \mu \mathrm{s}$ and amplitude of $10 \mathrm{~A}$. A homemade optocoupler is used as the wire position detector, it is based on fd-24k photodiode and provide $0.14 \mathrm{mV} / \mu \mathrm{m}$ gradient. Since the size of the undulator do not allow avoiding intersection of the main signal and reflections by elongation of the wire and the period of the undulator can be changed during operation of the FEL it was decided to use dampers based on oil drops, see [6], placed on the ridges of an automated pantograph.

Several trial launches of the system were performed, see example in Fig. 2.

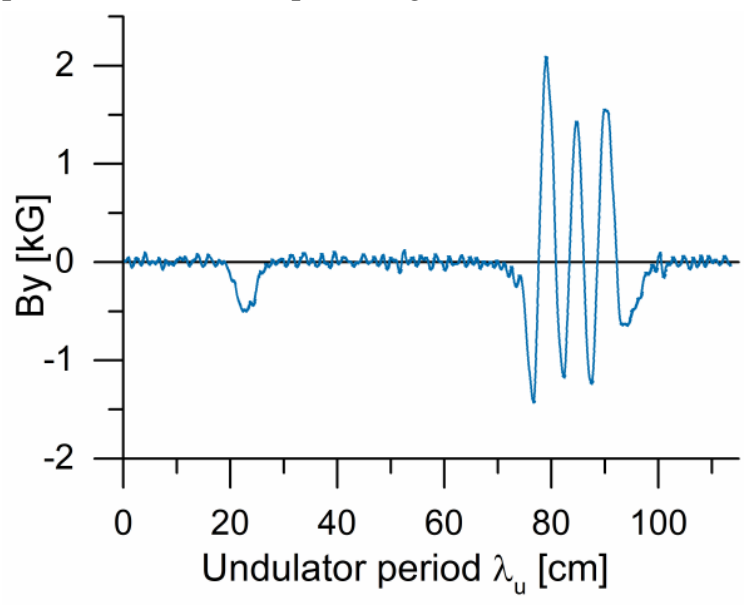

Fig. 2. Test measurement

The field distribution was reconstructed from the measured first integral data by differentiating, no filter was applied.

\section{Discussion and conclusion}

The described experimental setup is under active development now. Test measurements of reference magnet and VPU short prototype were conducted, see Fig. 2, and obtained results indicate that detector sensitivity should be improved in order to obtain larger signal to noise ratio.

The task to commission a pulsed wire measurements setup over $10 \mathrm{~m}$ long has not been challenged yet. It is yet to be determined whether complete compensation of signal distortion effects e.g. wire sagging influence, attenuation and dispersion is possible during postprocessing. Alternatively, complementary components, like wire supports and additional detector, could be applied to minimize the influence of distortion effects.

Currently authors attempt to increase the sensitivity of the detector and suppress impact from external noises in order to acquire clean data.

\section{Acknowledgements}

The work was supported by the Russian Science Foundation (project No. 14-50-00080).

\section{References}

1. Vinokurov N.A., Shevchenko O.A., Tcheskidov V.G. Variable-period permanent magnet undulators // Phys. Rev. Spec. Top. - Accel. Beams. 2011. V. 14. No. 4. P. 040701.

2. Davidyuk I.V., Shevchenko O.A., Tcheskidov V.G., Vinokurov N.A. Modeling and designing of variable-period and variable-pole-number undulator // Phys. Rev. Accel. Beams. 2016. V. 19. No. 2. P. 020701.

3. Warren R.W. Limitations on the use of the pulsedwire field measuring technique // Nucl. Instruments Methods Phys. Res. Sect. A Accel. Spectrometers, Detect. Assoc. Equip. 1988. V. 272. No. 1-2. P. 257-263.

4. Fan T.C., Lin F.Y., Hwang C.S., Hsu I.C. Pulsed wire magnetic field measurements on undulator U10P // PACS2001. Proceedings of the 2001 Particle Accelerator Conference (Cat. No.01CH37268). Chicago: IEEE, 2001. P. 2775-2777.

5. Kumar V., Mishra G. Analysis of pulsed wire method for field integral measurements in undulators // Pramana. 2010. V. 74. No. 5. P. 743-753.

6. Varfolomeev A.A. et al. Wire method for magnetic field measurements in long undulators // Nucl. Instruments Methods Phys. Res. Sect. A Accel. Spectrometers, Detect. Assoc. Equip. 1995. V. 359. No. 1-2. P. 93-96. 